\title{
Positronium Hyperfine Splitting
}

\author{
Akira Miyazaki, \\ Department of Physics, Graduate School of Science \\ and International Center for Elementary Particle Physics (ICEPP), \\ The University of Tokyo, 7-3-1 Hongo, Bunkyo-ku, Tokyo, 133-0033, Japan \\ miyazaki@icepp.s.u-tokyo.ac.jp
}

\begin{abstract}
Positronium is an ideal system for the research of QED in the bound state. The hyperfine splitting of positronium (Ps-HFS: about $203 \mathrm{GHz}$ ) is a good tool to test QED and also sensitive to new physics beyond the Standard Model. Previous experimental results show $3.9 \sigma(15 \mathrm{ppm})$ discrepancy from the QED O $\left(\alpha^{3} \ln 1 / \alpha\right)$ prediction. We point out probable common systematic errors in all previous experiments. I measure the PsHFS in two different ways. (1) A prototype run without RF system is described first. (2) I explain a new direct Ps-HFS measurement without static magnetic field. The present status of the optimization studies and current design of the experiment are described. We are now taking data of a test experiment for the observation of the direct transition.
\end{abstract}

\section{Introduction}

Positronium (Ps), the electron-positron bound state, is a purely leptonic system. The energy difference between ortho-positronium (o-Ps, ${ }^{3} S_{1}$ state) and parapositronium ( $p$-Ps, ${ }^{1} S_{0}$ state) 11 is called hyperfine splitting of positronium (PsHFS). It is a good target to study bound state QED precisely. The Ps-HFS value is approximately $203 \mathrm{GHz}(0.84 \mathrm{meV})$, which is significantly larger than hydrogen HFS $(1.4 \mathrm{GHz})$. About one third of this large value is contributed by a quantum oscillation as shown in Fig. [1: $o-\mathrm{Ps} \rightarrow \gamma^{*} \rightarrow o$-Ps 2 . Since some hypothetical particles, such as a milicharged particle, can participate in the quantum oscillation to shift Ps-HFS value, its precise measurement provides a probe into new physics beyond the Standard Model.

Measurements of the Ps-HFS have been performed in 70's and 80's [1, 2. The results were consistent with each other, and combined precision of $3.3 \mathrm{ppm}$ is obtained. They were consistent with $\mathrm{O}\left(\alpha^{2}\right)$ calculation of the QED available at that time. The corrections of $\mathrm{O}\left(\alpha^{3} \ln 1 / \alpha\right)$ have been calculated using NonRelativistic QED (NRQED) in 2000 [3]. The new prediction is $\Delta_{\mathrm{HFS}}^{\mathrm{th}}=203.39169(41) \mathrm{GHz}$, where the uncertainty is the unknown nonlogarithmic $\mathrm{O}\left(\alpha^{3}\right)$ term estimated in an analogous way to the HFS of muonium. This

\footnotetext{
${ }^{1}$ Although $p$-Ps decays mainly into two photons with lifetime of 125 ps, it takes $142 \mathrm{~ns}$ for $o$-Ps to decay. This is because $o$-Ps can only decay into three photons which is strongly suppressed by invariant matrix and kinematics. Two photon decay of $o$-Ps is forbidden by $\mathrm{C}$ conservation.

${ }^{2}$ Ortho-Ps has the same quantum number as a photon.
} 
calculated value differs from the measured value of $\Delta_{\mathrm{HFS}}^{\exp }=203.38865(67) \mathrm{GHz}$ by $3.9 \sigma$ as shown in Fig. 2. This discrepancy may be due to common systematic errors in the previous measurements or to new physics beyond the Standard Model.

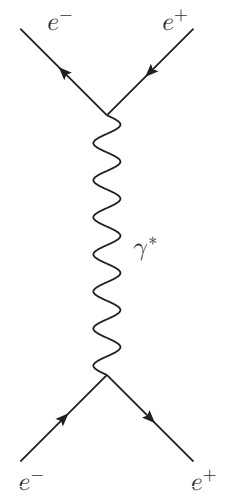

Figure 1: o-Ps contribution to Ps-HFS

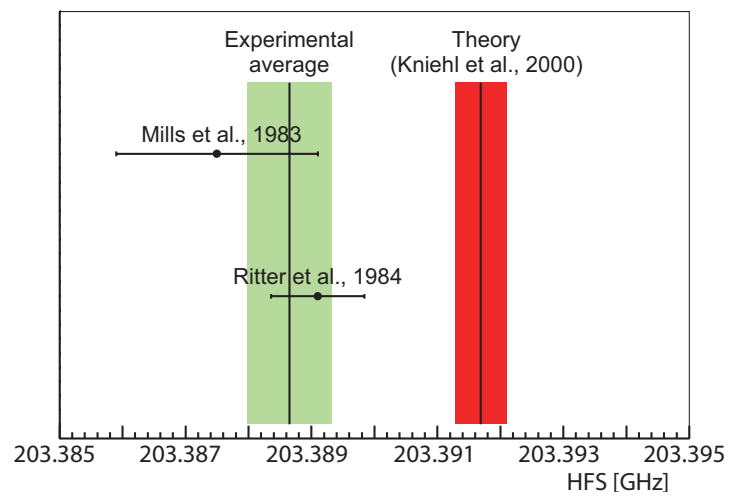

Figure 2: The discrepancy of Ps-HFS value

In all previous measurements, the Ps-HFS value was not directly measured, since $203 \mathrm{GHz}$ was too high to produce and control. Zeeman splitting of o-Ps has been measured instead. A static magnetic field makes Zeeman mixing between $m_{z}=0$ spin state of $o$-Ps and $p$-Ps. As a result, the energy level of $m_{z}=0$ state of $o$-Ps becomes higher than $m_{z}= \pm 1$ state. This Zeeman splitting, which is approximately proportional to Ps-HFS, is a few $\mathrm{GHz}$ frequency under about 1 Tesla magnetic field. Static magnetic field is applied in RF cavities where positronium is produced. Zeeman transition from $o$-Ps of $m_{z}= \pm 1$ to $o$-Ps of $m_{z}=0$ has been observed.

We point out the following three possibilities as the common systematic errors in these indirect measurements.

1. They may underestimate the non-uniformity of the magnetic field.

2. The unthermalized $o$-Ps contribution can result in an underestimation of the material effect.

3. RF systems to cause the transition might not be sufficiently stable.

Direct measurement of Ps-HFS without any magnetic fields is a main topic. I show the status of a prototype experiment in section 3 . It is completely free from the systematic errors of the magnetic field. A proposal about the second possibility is summarized by A. Ishida, et al. 4. The third point is also promising. The experiment with quantum oscillation instead of the RF source is performed. I report the result of this experiment in section 2. 


\section{Measurement with quantum oscillation}

We used a completely different method from previous one (i.e. without a RF source). This method is theoretically proposed by V.G. Baryshevsky et al. [5], in which a quantum oscillation between two Zeeman energy levels of $o$ $\mathrm{Ps}_{\mathrm{s}}$ is measured in a static magnetic field. Positrons emitted from a $\beta^{+}$source are polarized in the direction of their momentum due to parity violation in the weak interaction. Consequently, the resulting $o$-Ps is also

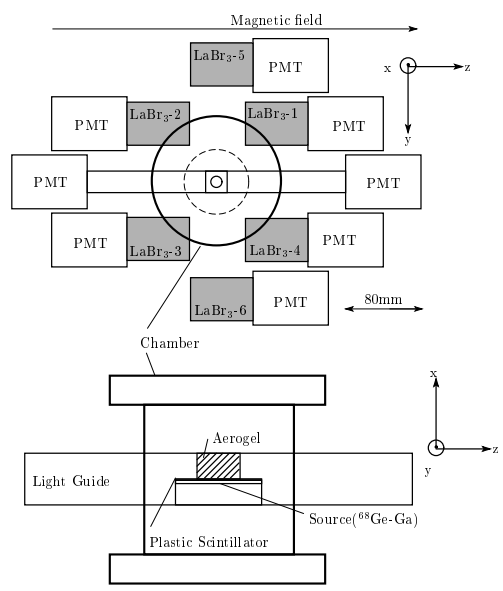

Figure 3: Schematic diagram of the setup highly polarized. This $o-$

Ps is a superimposed state of above two energy eigenstates of $o$-Ps in a static magnetic field. When a perpendicular magnetic field of about $0.1 \mathrm{~T}$ is induced to the polarization axis of this $o$-Ps, this quantum oscillation can be detected as an observable oscillation in timing spectra of $o$-Ps decay.

This approach is quite different from the previous all experiments. It is free from systematic errors originating from the high power light source and the RF cavity with high Q-value. Instead of them, precise measuring technique of decay curve is crucial. Especially, time-to-digital converter (TDC) with high performance is essential for precise measurement of the timing spectrum. Therefore, these two different approaches are complementary. Both experimental approaches are necessary to understand the discrepancy.

Figures 3 show a schematic diagram of the experimental setup of quantum oscillation. The upper figure shows the entire experimental setup. The magnetic field direction is along the $z$-axis. The $\mathrm{LaBr}_{3}(\mathrm{Ce})$ scintillators are placed in the $y z$-plane. They detect $\gamma$-rays with high energy resolution of $4.0 \%$ (FWHM) at $511 \mathrm{keV}$ and high timing resolution of $200 \mathrm{ps}$ (FWHM). The direction of the $\beta^{+}$emitted from the ${ }^{68} \mathrm{Ge}-\mathrm{Ga}$ source is along the $x$-axis. The bold circle is a vacuum chamber. The coordinate system is also shown. The lower figure is a magnified view of the vacuum chamber, in which the ${ }^{68} \mathrm{Ge}-\mathrm{Ga}$ source, the thin plastic scintillator and the silica aerogel are located. This plastic scintillator tags positrons, which go into the silica aerogel. Positronium is formed in this aerogel to decay into $\gamma$-rays. The signal from plastic scintillator comes at approximately the formation time of positronium. The time of the signal from $\mathrm{LaBr}_{3}$ is the decay time. We took a delayed coinsidence between them to make a decay curve of positornium. The time is measured with direct clock TDCs ( $5 \mathrm{GHz}$ : timing resolution of $200 \mathrm{psec}$ ). These TDCs have excellent integral and differential linearities.

Figures 4 show the measured time spectra at a magnetic value of $100 \mathrm{mT}$ (left 
figure) and $135 \mathrm{mT}$ (right figure). In both figures, the data points are plotted with error bars while the solid lines show the best fit results. A result of a prototype experiment is obtained as $\Delta_{\mathrm{HFS}}^{\exp }=203.324 \pm 0.039$ (stat.) \pm 0.015 (sys.) GHz. The accuracy is $200 \mathrm{ppm}$, which is an improvement by a factor of 90 over the previous experiment which used the similar method [6]. This result is consistent with both theoretical calculations and previous precision measurements of transition. However, we showed that we can improve this result to compete the most precise measurement with some simple improvements of our detection system. (in this paper [7])
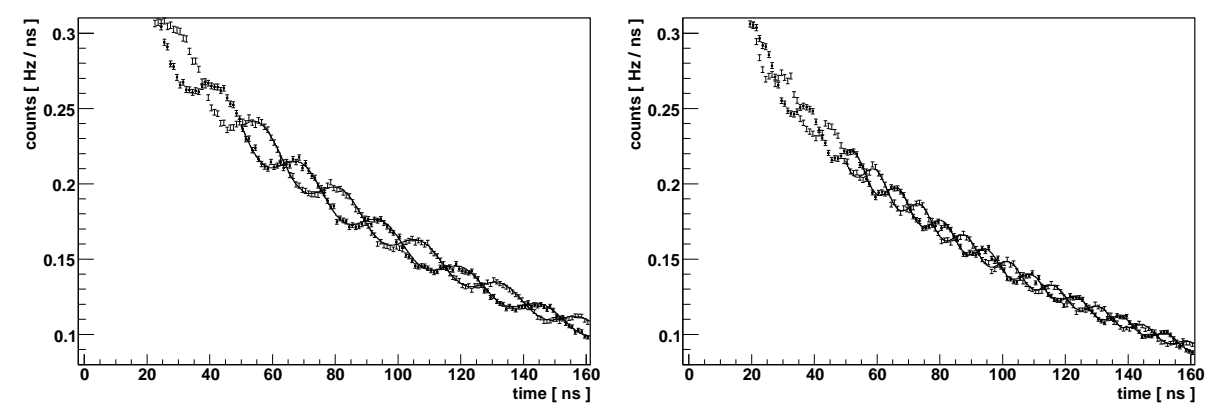

Figure 4: Timng spectra of the decay curve of $o$-Ps

\section{Direct measurement of Ps-HFS}

We are planing to directly measure the HFS transition, which does not need a static magnetic field. It is thus free from the systematic error from the field mentioned in section 1. However, the direct transition from o-Ps to $p$-Ps has very small probability of $3 \times 10^{-8} \mathrm{~s}^{-1}$, since this transition is $M 1$ transition, and the Ps-HFs is extremely large. Therefore, a powerful radiational field of $203 \mathrm{GHz}$ is essential so as to stimulate the direct transition.

The frequency of $203 \mathrm{GHz}$ is just intermediate between optical light and radiowave. There was no high power light source for spectroscopy in sub- $\mathrm{THz}$ region. We are developing a new light system in this region. A frequency tunable radiational source is necessary to measure a whole shape of the resonance curve 3. Our first target is to just observe the direct transition from o-Ps to $p$-Ps 4 . In order to accomplish this goal, we are developing following three new optical devices.

1. Sub-THz to THz light source called gyrotron,

2. Efficient transportation system of mode converter,

3. Parallel etalon with high-finesse called Fabry-Pérot cavity.

These are explained from the next subsection.

\footnotetext{
${ }^{3} \mathrm{RF}$ system in previous precise indirect measurements of $3 \mathrm{GHz}$ was not tunable, neither They changed the strength of a magnetic field to shift the Zeeman splitting to tune effectively the resonance frequency of Zeeman transition.

${ }^{4}$ This can be detected as an increase of two photon-decay ratio.
} 


\subsection{Gyrotron}
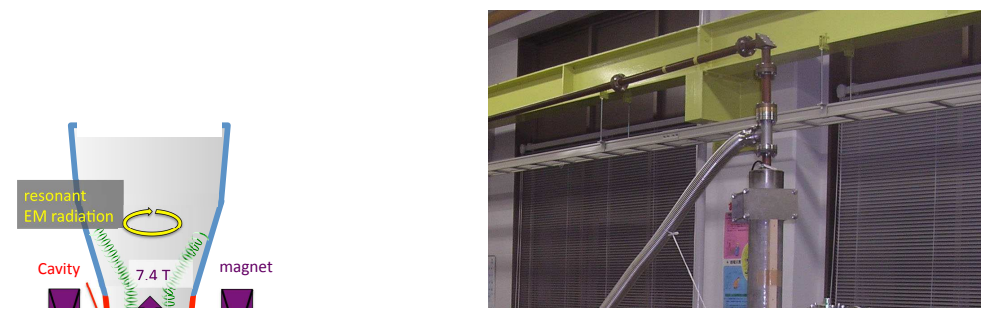

The gyrotron [8] is a novel high power light source for sub- $\mathrm{THz}$ to $\mathrm{THz}$ frequency region. It has been developed in the field of nuclear fusion. The structure of gyrotrons is shown in Fig. 5. The electrons are emitted from the DC electron gun, concentrated and rotated as cyclotron motion in the superconducting magnet. The cyclotron frequency $f_{c}$ is given by

$$
f_{c}=\frac{e B}{2 \pi m_{0} \gamma},
$$

where $B$ is the magnetic field strength, $m_{0}$ is the electron rest mass, and $\gamma$ is the relativistic factor of the electron. A cavity is placed at the maximum magnetic field in which resonant

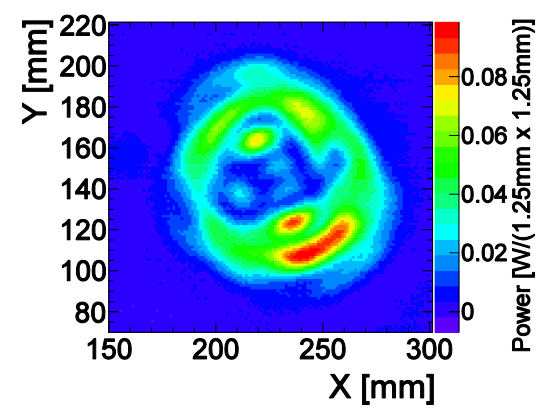

Figure 7: Power profile frequency is given by

$$
f=\frac{1}{2 \pi} \sqrt{\left(\frac{\chi_{m n}}{R}\right)^{2}+\left(\frac{l \pi}{L}\right)^{2}},
$$

where $R, L$ are radius and length of the cavity, respectively. $l$ is an index of longitudinal mode. $m, n$ are indices of transverse mode. $\chi_{m n}$ is a root of differential bessel function. This cavity frequency is tuned just to the cyclotron frequency to enhance the monochromatic light. The electrons stimulate cyclotron resonance maser in the cavity. The produced coherent photons are guided to the output port through the window, while electrons are dumped at a collector.

We developed a gyrotron operating at $f_{c}=203 \mathrm{GHz}$ with $B=7.364$ Tesla, $\gamma \sim 1.02$, which is shown in Fig. 6. The stable power of $300 \mathrm{~W}$ is obtained at 
the output window of gyrotron. The frequency width, which is determined by $B$ uniformity and $\gamma$ spread by thermal distribution of electrons, is expected to be less than $1 \mathrm{MHz}$. It is narrow enough to control the resonance at the FabryPérot cavity. Measured result with a similar gyrotron shows the frequency width is less than $10 \mathrm{kHz}$ [9. Although the frequency can be tuned by changing the $\gamma$ factor with different acceleration of electrons, the tuning range is limited by the resonant width of the cavity to several hundreds of megahertz.

Figure 7 shows the power profile of the radiation at taken with an infrared (IR) camera. The profile has a circular polarization called TE03 mode. Unfortunately, the mode inside Fabry-Pérot cavity is a linearly polarized gaussian mode. Therefore, the original gyrotron output cannot couple with Fabry-Pérot cavity. That's why a mode conversion is necessary to use gyrotron power efficiently.

\subsection{Mode converter}

Transportation system is composed of three parabolic mirrors called M0, M1 and M2 as shown in Fig. 8. The first parabolic mirror M0 converts polarization from circular to linear. M1 and M2 simply change the shape of power distribution from bi-gaussian to gaussian. Then, plain mirror M3 reflects radiaion and introduces it into Fabry-Pérot cavity. The power distribution is successfully converted into gaussian-like mode. Coupling between input light and FabryPérot cavity is now about $60 \%$. However, the transformation efficiency is $30 \%$, because the output of the current gyrotron is not optimized. As a result, about $20 \%$ of the original radiation from gyrotron can resonate in the cavity.
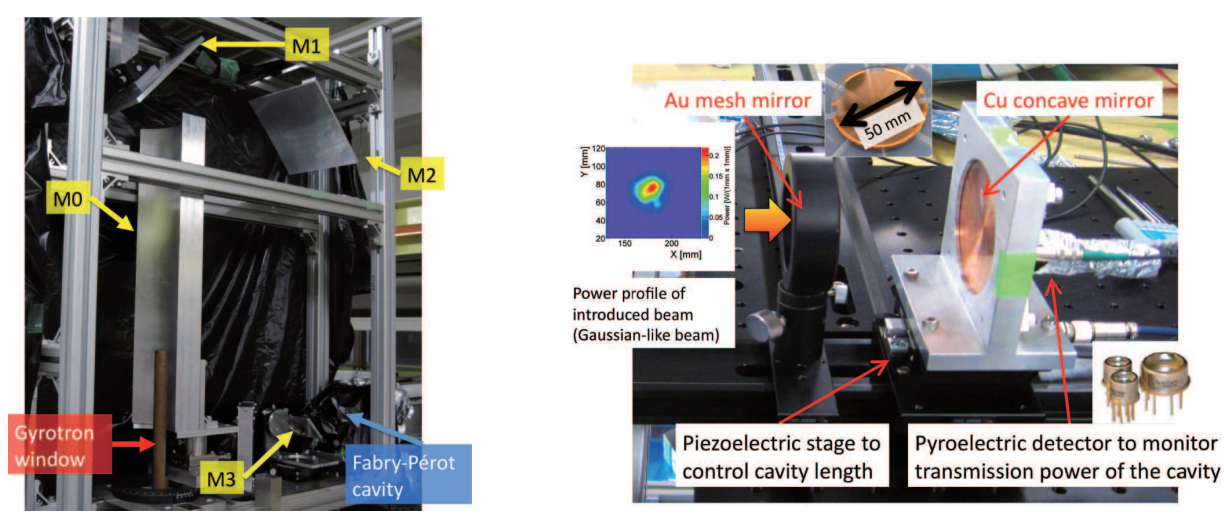

Figure 8: Transportation

Figure 9: Fabry-Pérot cavity

\subsection{Fabry-Pérot cavity}

Photons produced at the gyrotron are transported and accumulated in a cavity. Since $203 \mathrm{GHz}$ photons can be treated optically at the centimeter or larger size scale, we use a Fabry-Pérot cavity, which consists of two opposing mirrors to confine photons between them. Unlike RF cavities, the confinement in the Fabry-Pérot cavity is 1-dimensional while the other four sides are open as shown 
in Fig9 A golden mesh mirror is used on the input side of the cavity to introduce photons from gyrotron. A copper concave mirror is used on the other side.

The two most important characteristics of a cavity are finesse $\mathcal{F}$ and input coupling C. With the reflectivity of mesh mirror $R_{f}$ and concave mirror $R_{e}$, finesse is defined as

$$
\mathcal{F}=\frac{\pi\left(R_{f} R_{e}(1-A)\right)^{1 / 4}}{1-\left(R_{f} R_{e}(1-A)\right)^{1 / 2}},
$$

where $A$ is the medium loss inside the cavity. Round-trip times $N$ of photon in the cavity is given by

$$
N=\mathcal{F} / 2 \pi,
$$

Therefore finesse characterizes the capability of the cavity to store photons inside. To maximize the $\mathcal{F}$, power losses must be minimized. There are 3 types of loss, diffraction loss, medium loss and ohmic loss. With the confinement of photons by the concave mirror, diffraction loss is negligible in our cavity. Medium loss in ga: 5 is measured as about $0.1 \%$. Ohmic loss occurs at the mirrors, which is around $0.15 \%$ at the copper mirror and less than $1.0 \%$ at the mesh mirror.

Input coupling is the fraction of input power matched to the cavity mode. It is an important parameter to efficiently introduce photons into the cavity. In our cavity, the input coupling is determined by transmittance of the input mesh minnn

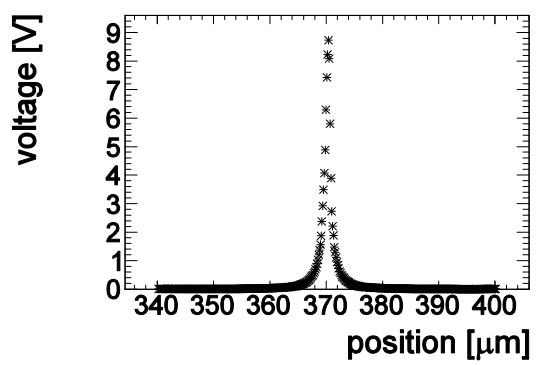

Figure 10: Transmission

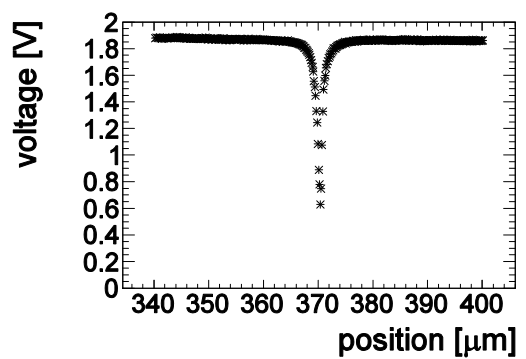

Figure 11: Reflection

Figure 9 shows the test setup, which is comprised of a mesh mirror on the mirror mount and a concave mirror on a piezo stage. Transmitted power is measured through a small hole on the concave mirror. On the other hand, reflected power is measured outside of the mesh mirror. When we shifted the cavity length precisely by the piezo stage, Breit-Wigner resonance was observed in the transmitted power monitor as shown in Fig. 10, Here, the horizontal axis is a position of the piezo stage, and the vertical axis is the output of power monitor.

Finesse is obtained from the width of the resonance $\Gamma$. Finesse is calculated by

$$
\mathcal{F}=\frac{\lambda / 2}{\Gamma},
$$

\footnotetext{
${ }^{5}$ Mixture of nitrogen $0.9 \mathrm{~atm}$ and isobutane $0.1 \mathrm{~atm}$.
} 
where $\lambda=1.47 \mathrm{~mm}$ is wavelegth of $203 \mathrm{GHz}$. We got finesse of about 650 , which is equivalent to 100 times round-trip according to Eq4

Figure 11] shows the measured reflection power. Input coupling is given by

$$
C=1-\frac{V_{\text {peak }}}{V_{\text {baseline }}} \text {. }
$$

Here, $V_{\text {peak }}$ is a voltage at peak decreasing from $V_{\text {baseline }}$, the voltage of the baseline of the reflected power. We achieved input coupling of $67 \%$. This large value is mainly due to a good mode conversion explained in the last subsection. The current status of the power is summarized in Table 1 The power of $10 \mathrm{~kW}$ is accumulated in the cavity.

Table 1: The summary of radiation power with our devices

\begin{tabular}{rrr}
\hline Device & Efficiency & power $(\mathrm{W})$ \\
\hline Gyrotron & 1 & 300 \\
Mode converter & 0.30 & 90 \\
Fabry-Pérot cavity & $0.60 \times 100 \times 2$ & about 10,000 \\
\hline
\end{tabular}

\subsection{Detection System}

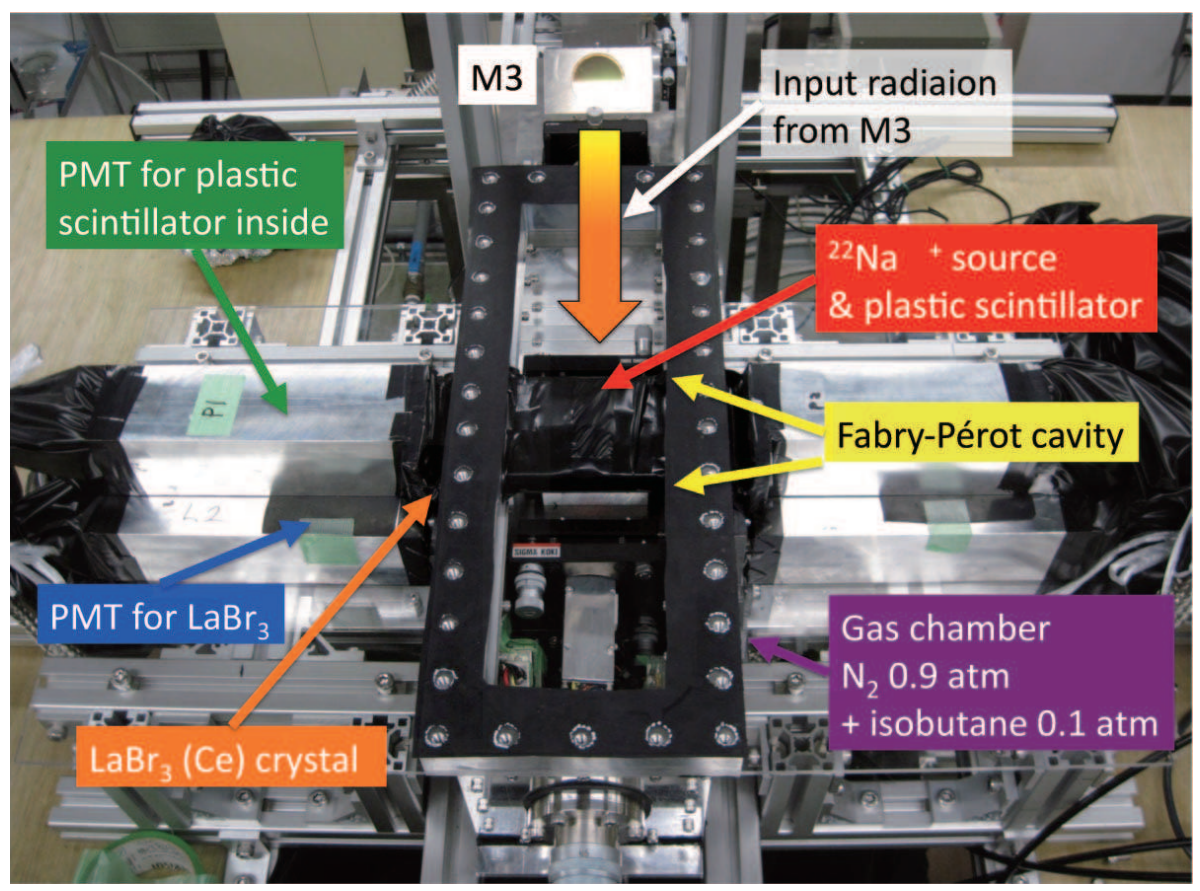

Figure 12: A photograph of gas chamber and detection system

Figure 12 shows a photograph of positronium production and signal detection system. Gyrotron power is introduced to the cavity via the mesh mirror, 
and accumulated inside Fabry-Pérot cavity. This cavity is placed inside a gas chamber filled with mixture gas of 0.9 atm nitrogen and 0.1 atm isobutane. The positron is emitted from $\beta^{+}$-source. The ${ }^{22} \mathrm{Na} \beta^{+}$-source is located $20 \mathrm{~mm}$ above the cavity. In order to generate start timing, the emitted positrons pass through a $\beta$-tag scintillator, with thickness of $100 \mu \mathrm{m}$. The signal from plastic scintillator transits through light guide made of acryl, to reach photomultiplier (PMT). Such kind of $\beta$-tagging system was also used in the experiment with quantum oscillation explained in section 2 .

A lead collimator, with thickness of $10 \mathrm{~mm}$, is placed under the plastic scintillator so as to select the positrons which go into the cavity. It also works as a shield to protect $\operatorname{LaBr}_{3}(\mathrm{Ce})$ scintillators from accidental photons 6 .

Positron forms positronium with an electron in the gas. Para-positronium annihilates into two $511 \mathrm{keV}$ photons immediately, while o-Ps remains with lifetime of $142 \mathrm{~ns}$ to decay into three photons, whose energy are continuous and less than $511 \mathrm{keV}$. A signal of the transition from $o$-Ps to $p$-Ps under $203 \mathrm{GHz}$ is a delayed-two-photon event. Four $\mathrm{LaBr}_{3}$ scintillators surround the chamber to detect photons. Two photon-decay can be easily separated from three photondecay with this energy information. The $\mathrm{LaBr}_{3}$ scintillators also have good timing resolution to separate delayed events (i.e. signal of transition) from prompt events 7 . to improve signal to background ratio significantly 8 .

The signal collection efficiency and background rates were estimated using Monte Carlo simulation (GEANT4). There are three major background processes. The first one is an three $\gamma$ contamination from $o$-Ps. The second one is a pick-off background. A positron in $o$-Ps interacts with a electron in a matter 9 only to annihilate into two photons. This process is called pick-off annihilation, and becomes background in our measurement. The last one is an accidental pileup process. In order to eliminate these backgrounds, we selected back-toback signal in $\mathrm{LaBr}_{3}$ scintillator and imposed condition that smeared energy deposit is $511 \mathrm{keV} \pm 3 \sigma$.

The obtained power of $10 \mathrm{~kW}$ is used for the simulation. Figure 13 shows an expected spectra for one month of data taking. The estimated rate is also summarized in Table 2. In this table, "ON" means the signal under $203 \mathrm{GHz}$ radiation while "OFF" means that without radiation 10 . The main background is three $\gamma$ contamination. And the other two backgrounds are the same size of the signal. We can clearly see the transition within one month.

\footnotetext{
${ }^{6}$ They are mainly $1275 \mathrm{keV}$ and $511 \mathrm{keV}$ photons emitted around the source.

7 Almost all the prompt events are two photon-decay.

8 In case of the power of $10 \mathrm{~kW}$ in the cavity, $\mathrm{S} / \mathrm{N}$ is estimated to be improved 16 times, when a timing window is imposed from 50 to $250 \mathrm{~ns}$ in decay curve.

${ }^{9}$ In this case nitrogen and isobutane.

${ }^{10}$ In Table 2, total $(\mathrm{ON})$ - total $(\mathrm{OFF})$ is not equal to signal. Because, the background events associated with $o$-Ps decreases under high power resonance radiation (i.e. ON). A Part of $o$-Ps transits into $p$-Ps to decay earlier than the timing window. As a result, total $(\mathrm{ON})$ total (OFF) becomes less than expected signal rate.
} 
Table 2: The summary of signal estimation

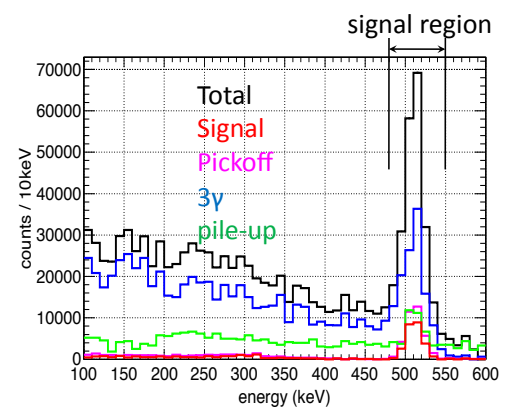

\begin{tabular}{cc}
\hline process & rate $(\mathrm{mHz})$ \\
\hline signal & 63 \\
pickoff & 93 \\
$3 \gamma$ & 300 \\
pileup & 100 \\
total $(\mathrm{ON})$ & 560 \\
total $(\mathrm{OFF})$ & 530 \\
\hline
\end{tabular}

Figure 13: Signal estimation

\section{Summary}

There is a large discrepancy between theory and experiment in Ps-HFS value. We suspect some common systematic errors in previous experiments. The prototype experiments without RF system was already performed. It was a complementary method against previous experiments, and the accuracy of $200 \mathrm{ppm}$ was obtained. We are now tackling the direct measurement without any magnetic fields. It is the first trial for sub- $\mathrm{THz}$ spectroscopy with $M 1$ transition. We have developed a high power $203 \mathrm{GHz}$ radiation source called gyrotron, mode converter and Fabry-Pérot cavity. Monte Carlo simulation of the detection system shows that the observation of Ps-HFS is feasible. We are now taking data of a test experiment for the direct transition from $o$-Ps to $p$-Ps. The signal is expected to be observed in a month.

These experiments are collaborated with Y. Sasaki, A. Ishida, T. Yamazaki, T. Suehara, T. Namba, S. Asai, T. kobayashi, H. Saito, M. Yoshida, K. Tanaka, M. Ikeno, A. Yamamoto, T. Idehara, I. Ogawa, Y. rushizaki and S. Sabchevski.

\section{References}

[1] A. P. Mills, Jr. et al. Phys. Rev. Lett. 34 (1975) 246; A. P. Mills, Jr. et al. Phys. Rev. A 27 (1983) 262.

[2] M. W. Ritter, et al. Phys. Rev. A 30 (1984) 1331.

[3] B. A. Kniehl and A. A. Penin, Phys. Rev. Lett. 85 (2000) 5094.

[4] A. Ishida, G. Akimoto, Y. Sasaki, et al. arXiv:1004.5555 (2010).

[5] V. G. Baryshevsky et al. J. Phys. B 22 (1989) 2835.

[6] S. Fan, et al. Phys. Lett. A 216 (1996) 129.

[7] Y. Sasaki, A. Miyazaki, et al. arXiv:1002.4567 (2010), accepted by Phys. Lett. $B$.

[8] T. Idehara, et al. IEEE Trans. Plasma Sci. 27 (1999) 340.

[9] T. Idehara, et al. Appl. Magn. Reson. 34 (2008) 265. 